\title{
Agrarrechtliche Weltenrettung oder Überforderung? \\ Von der Methodik der Integration außerlandwirtschaftlicher Ansprüche ins europäische Agrarrecht
}

\section{Einleitung}

Das europäische Agrarrecht fußt bekanntlich auf den Art. 38 bis 44 AEUV und verfügt insofern über einen offenen und relativ schlanken Handlungsauftrag. Die in Art. 39 AEUV genannten fünf klassische Ziele - Produktivitätssteigerung, Gewährleistung einer angemessenen Lebenshaltung der landwirtschaftlichen Bevölkerung, Stabilisierung der Märkte, Sicherstellung der Versorgung, Belieferung der Verbraucher zu angemessenen Preisen - sind mittels einer der drei Instrumente, die Art. 40 Abs. 1 AEUV zur Verfügung stellt, zu verfolgen. Das sind gemeinsame Wettbewerbsregeln, eine bindende Koordinierung der verschiedenen einzelstaatlichen Marktordnungen sowie eine europäische Marktordnung. Schon früh wurde die Option 3 gewählt, da nur eine Europäische Marktorganisation am Beginn der Gemeinsamen Agrarpolitik (GAP) als ausreichend geeignet eingestuft wurde, die Ziele und da insbesondere die Behebung der Mangellage nach dem Zweiten Weltkrieg zu erreichen.

Auf dieser Grundlage bildete sich in der Folge das europäische Agrarrecht aus, das in Umfang und Detaillierungsgrad wohl beispiellos in-

\footnotetext{
* Universität Luzern, Schweiz.
} 
nerhalb des Europarechts dasteht ${ }^{1}$. Dabei wurde die thematische Basis kontinuierlich ausgeweitet, wobei einerseits die agrarpolitische Agenda selbst beständig weiterentwickelt wurde, andererseits andere Politiken mehr oder weniger integriert wurden. Der Beitrag versucht zunächst in groben Umrissen die wichtigsten dieser Ausweitungen nachzuzeichnen (Kapitel 2) und daran anschließend die Instrumente, mit denen diesen jeweils neuen Materien begegnet wird, zu systematisieren (Kapitel 3) und zu analysieren (Kapitel 4).

\section{Erweiterungen}

Gemessen an der Ausgangslage zur Zeit der Konferenz von Stresa vom Juli 1958, die dem Auftrag des ex-Art. 43 Abs. 1 EWGV entsprechend die Grundlinien einer GAP erarbeitete ${ }^{2}$, mag die heutige thematische Breite erstaunen. Gleichwohl wurden die primärrechtlichen Grundlagen nicht ohne Grund als Rahmenregelung konzipiert, um der konkreten politischen Ausgestaltung je nach Betriebszweigen und -formen, geographischen Regionen und Ländern, Themen und Zeiten ausreichend Spielraum zu erhalten ${ }^{3}$. Dieses primärrechtliche Steuerungsdefizit hat sich denn auch kaum nachteilig ausgewirkt und die hier beschriebenen Transformationen erst ermöglicht.

\subsection{Marktordnung}

Der eigentliche juristische Start der GAP datiert ins Jahr 1962 mit den ersten Verordnungen zur schrittweisen Einrichtung der gemeinsamen Marktorganisationen $(\mathrm{GMO})^{4}$, die für die meisten Produkte bis 1970 abgeschlossen

1 J. Schwarze, Europäisches Verwaltungsrecht, Baden-Baden 2005, S. 380.

2 Vgl. R. Norer, Die Zukunft der Gemeinsamen Agrarpolitik: Europarechtlicher Trendsetter oder Expertenhochburg?, in: R. Norer, G. Holzer (Hrsg.), Agrarrecht Jahrbuch, Wien 2020, S. 217; O. Gottsmann, Art. 43 EWGV Rz. 2, in: H. von der Groeben, H. von Boeckh, J. Thiesing, C.D. Ehlermann (Hrsg.), Kommentar zum EWG-Vertrag, Baden-Baden 1983.

3 Siehe R. Norer, Art. 38 AEUV Rz. 4, in: M. Pechstein, C. Nowak, U. Häde (Hrsg.), Frankfurter Kommentar EUV, GRC und AEUV, Band II, Tübingen 2017, m.w.N.

${ }^{4}$ Dazu VO (EWG) Nr. 25/1962 über die Finanzierung der gemeinsamen Agrarpolitik, AB1. 1962, Nr. 30/991; VO (EWG) Nr. 26/1962 zur Anwendung bestimmter Wettbewerbsregeln auf die Produktion landwirtschaftlicher Erzeugnisse und den Handel mit diesen Erzeugnissen, ABl. 1962, Nr. 30/993. 
werden konnten ${ }^{5}$. Diese Regelwerke präsentierten sich ausschließlich als von genuin agrarwirtschaftsrechtlichem Charakter, umfassend insbesondere Preis- und Handelsregelungen, Interventionsmaßnahmen, Einfuhrlizenzen und Erstattungen, dazu noch die Grundregeln für die Finanzierung der GAP. Nicht mehr und nicht weniger.

Die weitere Entwicklung dieses Kernbestands des europäischen Agrarrechts war dann durch die Fehlentwicklungen der 1980er und beginnenden 1990er Jahre gekennzeichnet, die sich in einer Überschussproduktion (die sprichwörtlichen Milchseen und Butterberge) und damit verbundenen extremen Finanzlasten manifestierten. Erst die bis heute wegweisende GAP-Reform 1992 (MacSharry-Reform $)^{6}$ mit ihrer Abkehr von der Preisgarantie hin zu direkten flächen- und ertragsorientierten Einkommensbeihilfen transformierte das ökonomische Instrumentarium nachhaltig.

Nach der Fortführung im Rahmen der „Agenda 2000“7 vermochte dann die Reform 2003 (Fischler-Reform $)^{8}$ mit der Einführung der produktionsunabhängigen einheitlichen Betriebsprämie einen weiteren radikalen Schritt zu machen, wobei sich diese Reformetappe in der Rückschau janusköpfig präsentiert: einerseits als Fortentwicklung der 1992 eingeleiteten Reformschritte, andererseits als Systembruch aufgrund der grundsätzlichen Entkoppelung der Direktzahlungen 9 .

Mit der Reform $2013^{10}$ wurde schließlich die Zuweisung von Zahlungsansprüchen aufgrund historischer Referenzwerte wieder aufgegeben und durch die Basisprämienregelung abgelöst. In der Folge kam es mit dem Auslaufen der Milchquoten 2015 und Zuckerquoten 2017 zur Aufgabe jahrzehntelanger zentraler agrarwirtschaftlicher Regelungssysteme, neu erschienen Sicherheitsklauseln und die Ausdehnung der Anerkennung von Erzeugerorganisationen und Branchenverbänden auf alle Sektoren auf der agrarrechtlichen Bühne.

${ }^{5}$ Bspw. VO Nr. 120/67/EWG über die gemeinsame Marktorganisation für Getreide, AB1. 1967, L 117/2269; VO Nr. 121/67/EWG über die gemeinsame Marktorganisation für Schweinefleisch, ABl. 1967, L 117/2283; VO (EWG) Nr. 805/68 über die gemeinsame Marktorganisation für Rindfleisch, ABl. 1968, L 121/67. Dazu VO (EWG) Nr. 729/70 über die Finanzierung der gemeinsamen Agrarpolitik, ABl. 1970, L 94/13.

${ }^{6}$ VO (EWG) Nr. 1765/92 und 1766/92, ABl. 1992, L 181/12 ff.; VO (EWG) Nr. 2066/922080/92, AB1. 1992, L 215/49 ff.

7 VO (EG) Nr. 1251/1999-1259/1999, ABl. 1999, L 160/1 ff.; VO (EG) Nr. 1493/1999, ABl. 1999, L 179/1.

${ }^{8}$ VO (EG) Nr. 1782/2003-1788/2003, ABl. 2003, L 270/1 ff.; DurchführungsVO (EG) Nr. 795/2004 und 796/2004, AB1. 2004, L 141/1 ff.

${ }^{9}$ G. Eckhardt, Die Reform der GAP 2003 - Zwischenbilanz und Ausblick, in: R. Norer, G. Holzer (Hrsg.), Agrarrecht Jahrbuch, Wien - Graz 2010, S. 217.

${ }^{10}$ VO (EU) Nr. 1305/2013-1308/2013, AB1. 2013, L 347/487 ff. 


\subsection{Umweltschutz i.w.S.}

Ungeachtet diverser früherer Entwicklungen ${ }^{11}$ traten Aspekte einer eigenen Agrarumweltpolitik im Rahmen der GAP-Reform 1992 erstmals deutlich hervor. Der Gewährleistung einer extensiven, umweltgerechten Landwirtschaft dienten die im Rahmen der Verordnung (EWG) Nr. 2078/92 12 von den Mitgliedstaaten anzubietenden Agrarumweltprogramme. Dieser Rechtsrahmen wurde dann mit der Reform 1999 („Agenda 2000“) von einer flankierenden Maßnahme in die neu geschaffene 2. Säule der GAP aufgenommen ${ }^{13}$, wo sie bis heute ${ }^{14}$ - neben zahlreichen anderen, teilweise ebenfalls ökologisch ausgerichteten Maßnahmen - besteht. Dabei werden i.d.R. fünf- bis siebenjährige Verpflichtungen eingegangen, die auf die Erhaltung und Förderung notwendiger Änderungen landwirtschaftlicher Verfahren, die sich positiv auf die Umwelt auswirken, abzielen.

Mit der Agenda 2000 kam es aber auch erstmals zur Integration von Umweltzielen in die 1. Säule ${ }^{15}$. Unter dem Titel „Erfordernisse des Umweltschutzes" waren die Mitgliedstaaten gehalten geeignete Umweltmaßnahmen zu ergreifen, als welche Beihilfen für Umweltschutzverpflichtungen in der Landwirtschaft, allgemeine Umweltauflagen sowie spezifische Umweltauflagen als Voraussetzung für Direktzahlungen genannt wurden.

Die Reform 2003 verankerte dann das bis heute geltende Cross-Compliance-System, das die (volle) Zahlung der Direktbeihilfen an die Einhaltung bestimmter Vorschriften in Bezug auf landwirtschaftliche Flächen, Erzeugung und Tätigkeit bindet. Grundanforderungen an die Betriebsführung (GAB) hinsichtlich Umweltschutz, Lebensmittelsicherheit, Gesundheit von Mensch, Tier und Pflanzen, Tierschutz sowie die Erhaltung von Flächen in

${ }^{11}$ Siehe im Detail G. Queisner, Rahmenbedingungen für eine umweltverträgliche Landwirtschaft im Europarecht. Zugleich ein Beitrag zur Reform der GAP, Cross Compliance und Klimaschutz, Baden-Baden 2013, S. 113 ff; G. Holzer, Die neue ,Ökoarchitektur “ der GAP, in: R. Norer, G. Holzer (Hrsg.), Agrarrecht Jahrbuch, Wien - Graz 2015, S. 122 ff.

12 VO (EWG) Nr. 2078/92 für umweltgerechte und den natürlichen Lebensraum schützende landwirtschaftliche Produktionsverfahren, ABl. 1992, L 215/85.

${ }_{13}$ Art. 22 ff. VO (EG) Nr. $1257 / 99$ über die Förderung der Entwicklung des Ländlichen Raumes durch den Europäischen Ausrichtungs- und Garantiefonds für die Landwirtschaft (EAGFL), AB1. 1999, L 160/80.

${ }_{14}$ Art. 28 VO (EU) Nr. 1305/2013 über die Förderung der ländlichen Entwicklung durch den Europäischen Landwirtschaftsfonds für die Entwicklung des ländlichen Raums (ELER), AB1. 2013, L 347/487.

${ }^{15}$ Vgl. Art. 3 VO (EG) Nr. 1259/1999 zur Festlegung von Gemeinschaftsregeln für Direktzahlungen im Rahmen der Gemeinsamen Agrarpolitik, ABl. 1999, L 160/113. 
einem guten landwirtschaftlichen und ökologischen Zustand (GLÖZ) wurden in das Marktorganisationsrecht einbezogen ${ }^{16}$.

Mit der Reform 2013 kam es neben Modifikationen bei Cross Compliance ${ }^{17}$ zur zusätzlichen Einführung des sog. Greening, wonach $30 \%$ des verfügbaren nationalen Finanzrahmens an die Anwendung bestimmter nachhaltiger landwirtschaftlicher Praktiken geknüpft werden. Dabei wird ein Teil der Direktzahlungen von der Einhaltung dem Klima- und Umweltschutz förderlichen Landbewirtschaftungsmethoden abhängig gemacht ${ }^{18}$. Diese obligatorische Ökologisierungskomponente der Basisprämie stellt somit eine Entgeltzahlung für erbrachte ökologische Dienstleistungen dar ${ }^{19}$. Die Greening-Maßnahmen gehen über die Cross-Compliance-Anforderungen hinaus und umfassen auf Ackerflächen die Anbaudiversifizierung und Anlage von ökologischen Vorrangflächen oder ökologisch gleichwertige Maßnahmen sowie die Erhaltung von Dauergrünland.

Thematisch umfassen die angesprochenen Umweltschutzthemen mittlerweile eine beeindruckende Fülle von Themen.

\subsubsection{Umweltschutz i.e.S.}

Die in Anhang II VO 1306/2013 aufgeführten Cross-Compliance-Vorschriften aus dem Bereich „Umweltschutz“ umfassen drei Mindestanforderungen in Bezug auf den Boden und Kohlenstoffbestand im Rahmen des GLÖZ ${ }^{20}$ sowie Standards zu Pflanzenschutzmitteln ${ }^{21}$. Die Greening-Maßnahmen beinhalten auf Ackerflächen die Anbaudiversifizierung und Anlage von ökologischen Vorrangflächen oder ökologisch gleichwertige Maßnahmen sowie die Erhaltung von Dauergrünland ${ }^{22}$.

${ }^{16}$ Art. 3 ff. i.V.m. Anhang III und IV VO (EG) Nr. 1782/2003 mit gemeinsamen Regeln für Direktzahlungen im Rahmen der Gemeinsamen Agrarpolitik und mit bestimmten Stützungsregelungen für Inhaber landwirtschaftlicher Betriebe, ABl. 2003, L 270/1.

17 Art. 91 ff. i.V.m Anhang II VO (EU) 1306/2013 über die Finanzierung, die Verwaltung und das Kontrollsystem der Gemeinsamen Agrarpolitik, AB1. 2013, L 347/549.

${ }_{18}$ Art. 43 VO (EU) 1307/2013 mit Vorschriften über Direktzahlungen an Inhaber landwirtschaftlicher Betriebe im Rahmen von Stützungsregelungen der Gemeinsamen Agrarpolitik, AB1. 2013, L 347/608.

19 J. Martínez, Das Greening der Gemeinsamen Agrarpolitik, „Natur und Recht“2013, Nr. 35, S. 692.

${ }^{20}$ GLÖZ 4-6.

${ }^{21}$ GAB 10.

${ }^{22}$ Art. 43 ff. VO (EU) 1307/2013. 
In der ländlichen Entwicklung bezieht sich eine Priorität zur Gewährleistung der nachhaltigen Bewirtschaftung der natürlichen Ressourcen auf die Wiederherstellung, Erhaltung und Verbesserung der mit der Land- und Forstwirtschaft verbundenen Ökosysteme mit Schwerpunkten bei der biologischen Vielfalt, Wasserwirtschaft und Bodenbewirtschaftung ${ }^{23}$. Einschlägige Maßnahmen sind insbesondere die Agrarumweltmaßnahmen ${ }^{24}$ und der biologische Landbau ${ }^{25}$.

\subsubsection{Natur- und Biodiversitätsschutz}

Die in Anhang II VO 1306/2013 aufgeführten Cross-Compliance-Vorschriften aus dem Bereich „Biodiversität" beinhalten einzelne Bestimmungen der Vogelschutz- und Fauna-Flora-Habitat-Richtlinie sowie Anforderungen an die Landschaft und landschaftspflegerische Instandhaltungsmaßnahmen wie ein Verbot der Beseitigung von Landschaftselementen ${ }^{26}$.

Ausdrücklich auf den Naturschutz nimmt spätestens die Gewährung von Förderungen zur Entwicklung des ländlichen Raums seit 2005 Bezug, und zwar mit den Zahlungen im Rahmen von Natura 2000, um zusätzliche Kosten und Einkommensverluste auszugleichen, die aufgrund von Nachteilen im Zusammenhang mit der Vogelschutz- und Fauna-Flora-Habitat-Richtlinie entstehen ${ }^{27}$, sowie den Investitionen zum Erhalt der biologischen Vielfalt bei Arten und Lebensräumen ${ }^{28}$.

\subsubsection{Gewässerschutz}

Die in Anhang II VO 1306/2013 aufgeführten Cross-Compliance-Vorschriften aus dem Bereich „Wasser“ umfassen Art. 4 und 5 der Nitratricht$\operatorname{linie}^{29}$ sowie drei einschlägige von den Mitgliedstaaten festzulegende Mindeststandards für den Flächenzustand.

${ }^{23}$ Art. 4 lit. b und Art. 5 Z 4 VO (EU) Nr. 1305/2013.

${ }^{24}$ Art. 28 VO (EU) 1305/2013.

${ }^{25}$ Art. 29 VO (EU) 1305/2013.

${ }^{26}$ GAB 2-3, GLÖZ 7.

27 Art. 38 VO (EG) Nr. 1698/2005 über die Förderung der Entwicklung des ländlichen Raums durch den Europäischen Landwirtschaftsfonds für die Entwicklung des ländlichen Raums (ELER), AB1. 2005, L 277/1. Aktuell Art. 30 VO (EU) 1305/2013.

${ }^{28}$ Art. 17 Abs. 1 lit. d VO (EU) 1305/2013.

${ }^{29}$ GAB 1 und GLÖZ 1-3. 
In der 2. Säule werden Zahlungen im Rahmen der Wasserrahmenrichtlinie $^{30}$ vorgesehen, um zusätzliche Kosten und Einkommensverluste auszugleichen, die aufgrund von Nachteilen im Zusammenhang mit dieser Richtlinie entstehen.

\subsubsection{Tierschutz}

Die in Anhang II VO 1306/2013 aufgeführten Cross-Compliance-Vorschriften aus dem Bereich „Tierschutz" beziehen sich auf einzelne Artikel der drei europäischen Nutztierhaltungsvorschriften ${ }^{31}$.

Die Gewährung von Förderungen zur Entwicklung des ländlichen Raums beinhaltet insbesondere auch Tierschutzzahlungen ${ }^{32}$. Dabei umfasst werden freiwillige Tierschutzverpflichtungen, die über den verpflichtenden Standard von Cross Compliance hinausgehen.

\subsubsection{Klimaschutz ${ }^{33}$}

Während bei Cross Compliance der Anhang II VO 1306/2013 zwar den Klimawandel als ersten Bereich erwähnt, aber kaum klimaspezifische Maßnahmen vorsieht, werden mit dem zusätzlich eingeführten Greening $30 \%$ des verfügbaren nationalen Finanzrahmens an die Anwendung insbesondere dem Klimaschutz förderlicher Landbewirtschaftungsmethoden geknüpft ${ }^{34}$.

In der ländlichen Entwicklung bezieht sich eine Priorität zur Gewährleistung der nachhaltigen Bewirtschaftung der natürlichen Ressourcen auf die Förderung der Ressourceneffizienz und Unterstützung des Agrar-, Nahrungsmittel- und Forstsektors beim Übergang zu einer kohlenstoffarmen und klimaresilienten Wirtschaft mit Schwerpunkten bei der Wasser- und Energienutzung, erneuerbaren Energien, Verringerung der landwirtschaftlichen Treibhausgas- und Ammoniakemissionen sowie Kohlenstoff-Speicherung

30 Art. 30 VO (EU) 1305/2013.

31 GAB 11-13.

32 Art. 33 VO (EU) 1305/2013.

33 Zur Entwicklung siehe G. Holzer, Die neue Ökoarchitektur der GAP und ihr Beitrag zum Klimaschutz, in: R. Norer, G. Holzer (Hrsg.), Agrarrecht Jahrbuch, Wien - Graz 2019, S. $183 \mathrm{ff}$.

34 Art. 43 ff. VO (EU) 1307/2013. 
und -Bindung in der Land- und Forstwirtschaft ${ }^{35}$. Insbesondere eigene Klimamaßnahmen werden vorgesehen ${ }^{36}$.

\subsection{6. Ökopyramide}

Aktuell präsentiert sich das auf der Agrarkompetenz fußende System als komplexe Ökopyramide ${ }^{37}$, die sich stufenförmig von verpflichtend zu freiwillig zu erfüllenden Standards und vom umweltrelevanten Fachrecht über Cross Compliance und Greening bis zur ländlichen Entwicklung in schwindelerregende Höhen schraubt.

Auch wenn das ,greening the CAP“, also die Ausgestaltung des Fördersystems gemäß den Green-Box-Maßnahmen des WTO-Agrarabkommens $(\mathrm{AoA})^{38}$, die definitionsgemäß von den welthandelsrechtlichen Abbauverpflichtungen ausgenommen sind, am Anfang dieser Entwicklung gestanden haben mag, die heutige Ausdifferenziertheit der Maßnahmen geht weit über das völkerrechtlich Geforderte hinaus.

\subsection{Struktur- und Sozialpolitik}

Im Rahmen der, neben der Marktpolitik erst später angelaufenen gemeinsamen Agrarstrukturpolitik wurden 1972 soziostrukturelle Richtlinien verabschiedet und 1975 durch Maßnahmen zugunsten der Berggebiete und sonstigen benachteiligten Gebiete ergänzt ${ }^{39}$.

Während die flankierenden Maßnahmen der MacSharry-Reform Vorruhestandsregelungen ${ }^{40}$ vorsahen, wurde schließlich 1999 mit der „Agenda 2000“ die Förderung der Entwicklung des ländlichen Raums als gleichbe-

${ }^{35}$ Art. 4 lit. b und Art. 5 Z 5 VO (EU) 1305/2013.

${ }^{36}$ Art. 28 VO (EU) 1305/2013.

37 G. Holzer, Die neue „Ökoarchitektur “..., S. 133; idem, Die neue Ökoarchitektur der GAP..., S. 196.

$38 \mathrm{https} / /$ www.wto.org/english/docs_e/legal_e/14-ag_01_e.htm [besucht: 26.11.2021].

${ }_{39}$ RL 72/159/EWG über die Modernisierung der landwirtschaftlichen Betriebe, AB1. 1972, L 96/1; RL 72/160/EWG zur Förderung der Einstellung der landwirtschaftlichen Erwerbstätigkeit und der Verwendung der landwirtschaftlich genutzten Fläche für Zwecke der Strukturverbesserung, AB1. 1972, L 96/9; RL 72/161/EWG über die sozio-ökonomische Information und die berufliche Qualifikation der in der Landwirtschaft tätigen Personen, AB1. 1972, L 96/15; RL 75/268/EWG über die Landwirtschaft in Berggebieten und in bestimmten benachteiligten Gebieten, AB1. 1975, L 206/15.

${ }^{40} \mathrm{VO}$ (EWG) Nr. 2079/92 zur Einführung einer gemeinschaftlichen Beihilferegelung für den Vorruhestand in der Landwirtschaft, ABl. 1992, L 215/91. 
rechtigte 2. Säule der GAP geschaffen ${ }^{41}$. Diese legte den Rahmen für die gemeinschaftliche Förderung einer nachhaltigen Entwicklung und flankierte und ergänzte die anderen Instrumente der Gemeinsamen Agrarpolitik.

Heute verfolgt die 2. GAP-Säule u.a. die Erreichung einer ausgewogenen räumlichen Entwicklung der ländlichen Wirtschaft und der ländlichen Gemeinschaften, einschließlich der Schaffung und des Erhalts von Arbeitsplätzen. Eine der Prioritäten liegt dabei auf der Förderung der sozialen Inklusion, Armutsbekämpfung und wirtschaftlichen Entwicklung in ländlichen Gebieten mit Schwerpunkten bei Diversifizierung und Gründung kleiner Unternehmen und Schaffung von Arbeitsplätzen, Förderung der lokalen Entwicklung in ländlichen Gebieten sowie Förderung des Zugangs zu Informations- und Kommunikationstechnologien in ländlichen Gebieten $^{42}$. Einschlägige Maßnahmen sind insbesondere die Zahlungen für aus naturbedingten oder anderen spezifischen Gründen benachteiligte Gebiete ${ }^{43}$.

\subsection{Lebensmittelsicherheit}

Hier ist zunächst die Biologische Landwirtschaft, beginnend mit der EG-Öko-Verordnung 1991, zu erwähnen. Die gemeinsamen Regeln für Erzeugung, Kennzeichnung und Kontrolle des biologischen (ökologischen) Landbaus werden bereits seit 1992 in den Agrarumweltprogrammen gefördert ${ }^{44}$.

Heute ist die Lebensmittelsicherheit Bestandteil der Cross Compliance in Anhang II VO 1306/2013, bestehend aus zwei Bezugnahmen auf einschlägige Rechtsnormen ${ }^{45}$, ebenso aus den Bereichen Kennzeichnung und Registrierung von Tieren ${ }^{46}$ sowie Tierseuchen ${ }^{47}$.

\subsection{Energiepolitik}

Mit dem „Health Check“ 2009 wurde die ELER-Verordnung dahingehend geändert, dass die Mitgliedstaaten in ihren Entwicklungsprogrammen auch Vorhaben zum Klimawandel und zu erneuerbaren Energien aufnehmen ${ }^{48}$.

${ }^{41}$ VO (EG) $1257 / 1999$.

${ }^{42}$ Art. 4 lit. c und Art. 5 Z 6 VO (EU) 1305/2013.

${ }^{43}$ Art. 31 VO (EU) 1305/2013.

${ }^{44}$ Vgl. Art. 2 Abs. 1 lit. a VO (EWG) 2078/92 und aktuell Art. 29 VO (EU) 1305/2013.

${ }^{45}$ GAB 4-5.

${ }^{46}$ GAB 6-8.

${ }^{47}$ GAB 9.

${ }^{48}$ Art. 16a Abs. 1 lit. a und b VO (EG) 1698/2005, geändert durch VO (EG) Nr. 473/2009, AB1. 2009, L 144/3. 


\section{Instrumente}

Nach diesem kurzen Abriss sollen nun die Instrumente, mit denen diese thematische Ausweitung bis hin zur aktuellen GAP mit all ihren auch außerlandwirtschaftlichen Inhalten umgesetzt wird, untersucht werden.

Zunächst ist festzuhalten, dass integrierte Politiken dem Europarecht nicht nur nicht fremd sondern oftmals sogar ausdrücklich gewünscht sind. Paradebeispiel ist die Integrationsklausel des Art. 11 AEUV, wonach die Erfordernisse des Umweltschutzes bei der Festlegung und Durchführung der anderen Unionspolitiken und -maßnahmen insbesondere zur Förderung einer nachhaltigen Entwicklung einbezogen werden müssen. $\mathrm{Zu}$ diesen anderen Politiken zählt gemäß Art. 4 Abs. 2 lit. d AEUV gerade auch die Landwirtschaft. Auch wenn dieses Gebot in der Vorgängerregelung des Art. 6 EGV noch pointierter zum Ausdruck kam - demnach mussten die Erfordernisse des Umweltschutzes in die übrigen Politikbereiche «materiell integriert» werden - so ist die Verfolgung umweltpolitischer Ziele gerade auch durch die Agrarpolitik heute mehr denn je gefordert. Bereits 1992 nannte die erste Grundlage von Agrarumweltmaßnahmen in Art. 1 VO (EWG) Nr. 2078/92 als einen der angestrebten Zwecke zur „Verwirklichung der Ziele der Agrar- und Umweltpolitik der Gemeinschaft“ beizutragen. Die Verpflichtung zur Berücksichtigung von Umweltbelangen ist dabei nicht auf bestimmte Unionspolitiken beschränkt, sondern erfasst alle Tätigkeitsfelder der EU, neben der Landwirtschaft insbesondere auch die Verkehrs- und Energiepolitik ${ }^{49}$.

Methodisch lassen sich im Wesentlichen drei unterschiedliche Ansätze destillieren: eine Trennung der verschiedenen Rechtsmaterien (3.1.), deren Verknüpfung (3.2.) und deren - i.d.R. einseitige - Integration (3.3.).

\subsection{Trennung}

Kompetenzrechtlich und dogmatisch unproblematisch ist der Normalfall der Trennung der verschiedenen Rechtsgebiete und damit einhergehend auch der administrativen Zuständigkeiten. D.h. das europäische Umweltrecht, Verbraucherschutzrecht, Sozialrecht, Energierecht etc. wird auf Basis der einschlägigen primärrechtlichen Kompetenz erlassen (Art. 192, 169, 153, 194 AEUV), wenn nötig ins jeweils spezifische nationale Fachrecht

49 S. Heselhaus, Art. 11 AEUV Rz. 21, in: M. Pechstein, C. Nowak, U. Häde (Hrsg.), Frankfurter Kommentar..., Band II. 
umgesetzt und im Wege des meist vorgesehenen indirekten Vollzugs durch die nationalen Umwelt-, Verbraucherschutz-, Sozial- und Energiebehörden vollzogen.

\subsubsection{Allgemeine Normen}

Handelt es sich dabei um allgemeine Normen, gilt dieses Recht generell-abstrakt für jedermann und nicht nur für die Landwirtschaft. Landwirtschaftliche Bewirtschafter und Bewirtschafterinnen sind also wie alle anderen EU-Bürger und -Bürgerinnen daran gebunden.

Exemplarisch kann hier die unterste Stufe der Ökoarchitektur-Pyrami$\mathrm{de}^{50}$, das umweltrelevante Fachrecht angeführt werden. Diese allgemein verbindlichen Normen des Unions- und des nationalen Rechts beanspruchen allgemeine Geltung und können mittels fachspezifischer Sanktionen einheitlich durchgesetzt werden. Handelt es sich dabei allerdings um Richtlinien, eröffnen sich den nationalen Gesetzgebern teilweise große Spielräume mit der Folge unterschiedlicher Umweltstandards in den einzelnen Mitgliedstaaten, weshalb hier die Tendenz zu unmittelbar anwendbaren Verordnungen geht $t^{51}$.

Beispiele sind etwa die Grundwasserrichtlinie ${ }^{52}$, Wasserrahmen-Richtlinie $^{53}$, Umwelthaftungs-Richtlinie ${ }^{54}$ oder die Natura 2000-Richtlinien ${ }^{55}$.

\subsubsection{Spezifische Normen}

Ein zweiter, etwas anders gelagerter Fall ist jener, wo die ,andere“ Rechtsmaterie agrarspezifische Normen enthält, die dann - ausdrücklich oder konkludent - einzig auf die Landwirtschaft Anwendung finden ${ }^{56}$. Es

${ }^{50}$ G. Holzer, Die neue „Ökoarchitektur“..., S. 133; idem, Die neue Ökoarchitektur der GAP..., S. 196.

${ }^{51}$ So G. Holzer, G. Holzer, Die neue „Ökoarchitektur “..., S. 134 mit Beispielen.

${ }^{52}$ RL 2006/118/EG zum Schutz des Grundwassers vor Verschmutzung und Verschlechterung, ABl. 2006, L 372/19.

${ }^{53}$ RL 2000/60/EG zur Schaffung eines Ordnungsrahmens für Maßnahmen der Gemeinschaft im Bereich der Wasserpolitik, AB1. 2000, L 327/1.

${ }^{54}$ RL 2004/35/EG über Umwelthaftung zur Vermeidung und Sanierung von Umweltschäden, AB1. 2004, L 143/56.

${ }_{55}$ RL 2009/147/EG über die Erhaltung der wildlebenden Vogelarten, AB1. 2009, L 20/7; RL 92/43/EWG zur Erhaltung der natürlichen Lebensräume sowie der wildlebenden Tiere und Pflanzen, ABl. 1992, L 206/7.

${ }^{56}$ Diese Sondernormen können Ausnahmenormen (ius singulare) oder Spezialnormen (ius proprium) sein; dazu siehe Ch. Grimm, R. Norer, Agrarrecht, München 2015, S. 15. 
sind also (fast) ausschließlich nur landwirtschaftliche Bewirtschafter und Bewirtschafterinnen daran gebunden.

Exemplarisch für einzelne solcher spezifischen Normen in allgemeinen Regelwerken sei die Grundwasserrichtlinie erwähnt, in der insbesondere Grenzwerte für Nitrate und Wirkstoffe in Pflanzenschutzmitteln festgelegt $\operatorname{sind}^{57}$ oder die Umwelthaftungsrichtlinie, die als Tätigkeiten die Landwirtschaft anspricht $t^{58}$.

Exemplarisch für ganze Regelwerke solcher spezifischen Normen ist auf die Klärschlammrichtlinie ${ }^{59}$ und Nitratrichtlinie ${ }^{60}$ hinzuweisen, die beide den (exklusiven) Landwirtschaftsbezug bereits im Namen tragen ${ }^{61}$.

Aber auch hier gilt, dass diese Normen nicht unter der Agrarkompetenz erlassen worden sind und (grundsätzlich) nicht von den Landwirtschaftsbehörden vollzogen werden.

\subsection{Verknüpfung}

Ein zweiter, deutlich landwirtschaftsspezifischerer Weg besteht darin, die außerlandwirtschaftlichen Rechtsbereiche mit dem Agrarrecht zu verlinken. Damit erhalten bestimmte Normen des allgemeinen Fachrechts eine spezielle Bedeutung, die über ihre allgemeine Verbindlichkeit hinausgeht. Eine solche Verbindung ist in beide Richtungen denkbar, also die ,agrarfremde“ Norm enthält einen speziellen Verweis auf das Agrarrecht oder die Koppelung erfolgt in der agrarischen Norm selbst.

Während für den ersten Fall kein Beispiel ersichtlich ist, steht exemplarisch für den zweiten Fall im Umweltbereich die bereits beschriebene Cross Compliance ${ }^{62}$. Dort wird bei den Grundanforderungen an die Betriebsführung (GAB) an in Kraft stehende einzelne umweltrelevante Normen des Fachrechts angeknüpft. Diese müssen von Landwirten und Landwirtinnen dann nicht nur aufgrund ihrer allgemeinen Geltung eingehalten werden, sondern entfalten darüber hinaus im Agrarrecht für sie spezielle Wirkungen. So treten

\footnotetext{
57 Anhang I RL 2006/118/EG.

${ }^{58}$ Art. 3 Abs. 1 lit. a i.V.m. Anhang III Z 7c und 11 RL 2004/35/EG.

59 RL 86/278/EWG über den Schutz der Umwelt und insbesondere der Böden bei der Verwendung von Klärschlamm in der Landwirtschaft, AB1. 1986, L 181/6.

${ }^{60}$ RL 91/676/EWG zum Schutz der Gewässer vor Verunreinigung durch Nitrat aus landwirtschaftlichen Quellen, AB1. 1991, L 375/1.

${ }^{61}$ Überdies sind die Art. 4 und 5 RL 91/676/EWG Gegenstand des Cross-Compliance-Standards GAB 1; Anhang II VO (EU) 1306/2013.

${ }^{62}$ Art. 91 ff. i.V.m. Anhang II VO (EU) 1306/2013.
} 
im Fall einer Übertretung neben die fachrechtliche Sanktion zusätzlich noch Kürzungen der Direktzahlungen der 1. und 2. Säule ${ }^{63}$.

Hier wird die ,agrarfremde“ Norm unter der einschlägigen Kompetenznorm erlassen und von der jeweiligen Fachbehörde vollzogen, gleichzeitig aber erhält sie eine zweite Seite, indem sie aufgrund der in der Agrarkompetenz erlassenen Verlinkung zusätzlich auch von der Agrarbehörde exekutiert wird ${ }^{64}$.

\subsection{Integration}

Die dritte Möglichkeit kann darin gesehen werden, dass das Agrarrecht die sachfremde Materie selbst regelt. Dann handelt es sich um genuin agrarrechtliche Normen, die unter der Agrarkompetenz erlassen und ausschließlich von der Agrarverwaltung vollzogen werden.

Exemplarisch ist dabei das Greening. Hier werden im Agrarrecht klimaund umweltschutzfördernde Landbewirtschaftungsmethoden formuliert, deren Einhaltung bereits bei der Antragstellung im Mehrfachantrag dargelegt werden muss. Bei Verstößen kommt ein kompliziertes abgestuftes System von Kürzungen der Ökoprämie zur Anwendung ${ }^{65}$. Ein weiteres Beispiel sind die vielfältigen, auf europäischem und dieses umsetzendem nationalem Agrarrecht fußenden Maßnahmen der ländlichen Entwicklung, die freiwillig eingegangen werden und deren Standard über jenen von Cross Compliance und Greening hinausgehen muss.

\section{Analyse}

\subsection{Bewertung}

Die Trennung erweist sich als rechtlicher Normalfall. Die entsprechende Rechtsmaterie wird wie alle anderen allgemeinen Normen von der jeweils zuständigen (nicht agrarischen) Behörde vollzogen und allfällige Verstöße treffen entweder Landwirte und Landwirtinnen wie alle anderen Staatsbürger und Staatsbürgerinnen auch (allgemeine Norm) oder (fast) ausschließlich Landwirte und Landwirtinnen (spezifische Norm).

${ }^{63}$ Art. 97 ff. VO (EU) 1306/2013.

${ }^{64}$ F.-J. Peine, Verknüpfung der Beihilfen mit der Einhaltung von Umweltstandards - Konsequenzen, „Agrar- und Umweltrecht“ 2005, Beilage I, 12, spricht von einer Geltungsverstärkung.

${ }^{65}$ Art. 24-29 delegierte VO (EU) 640/2014 zur Ergänzung der VO (EU) 1306/2013, ABl. 2014, L $181 / 48$ 
Bei der Verknüpfung hat sich Cross Compliance trotz zahlreicher damit verbundener kritischer Rechtsfragen ${ }^{66}$ einen seither alle Reformetappen überdauernden Fixplatz behaupten können ${ }^{67}$. Im Gegenteil wird in der Literatur sogar eine Nachjustierung in Bezug auf eine stärkere Vereinheitlichung der Standards ${ }^{68}$ gefordert $^{69}$.

Hier sei aus der Reihe der Rechtsfragen nur auf das Kompetenzproblem eingegangen. Demnach führt Cross Compliance dazu, dass wesentliche Teile des Umweltrechts letztlich durch das Agrarrecht vollzogen werden und aufgrund der effizienten Förderungskontrollen i.d.R. deutlich an Steuerungswirkung gewinnen ${ }^{70}$. Dabei mutieren die Normen des Umweltrechts kraft Verlinkung für Zwecke der Horizontalen GAP-Verordnung gleichsam zu agrarrechtlichen Normen, die man gemäß rechtswissenschaftlicher Dogmatik $^{71}$ als sog. subsidiäres Agrarrecht bezeichnen kann. Die einschlägigen Normen verlassen ihren Fundort in der agrarfremden Materie nicht, bekommen aber kraft Verweises in landwirtschaftsspezifischen Zusammenhängen gleichsam eine zweite Rechtsnatur. Haben sie sich solcherart in diesem Zusammenhang zur Norm des Agrarrechts gewandelt, rechtfertigt es sich auch, dass sie von den Agrarbehörden vollzogen und sanktioniert werden. Dies auf Kosten des Agrarbudgets ${ }^{72}$.

Bei der Integration schließlich werden nicht ,agrarfremde“ Inhalte aus dem Fachrecht gleichsam ins Agrarrecht hereingeholt wie bei der Verlinkung, sondern im Agrarrecht selbst formuliert.

Hier kann sich die kompetenzrechtliche Frage stellen, ob solche Normen überhaupt rechtens vom Agrargesetzgeber erlassen werden dürfen. Der

${ }^{66}$ R. Norer, Rechtsfragen der EU-Agrarreform, Wien - Graz 2007, S. 107 ff.; für eine Zusammenschau der grundlegenden, in der Literatur aufgeworfenen Rechtsfragen siehe G. Holzer, Die neue ,Ökoarchitektur “..., S. $140 \mathrm{ff}$.

${ }^{67}$ G. Eckhardt (Die Reform der GAP 2003 ..., S. 103) spricht gar von einem Quantensprung, m.w.H.

${ }^{68}$ Diese Einheitlichkeit hängt derzeit v.a. von der Umsetzungspraxis der einzelnen Mitgliedstaaten ab, da die GAB-Verlinkung zumeist an EU-Richtlinien angesetzt, was aufgrund der unterschiedlichen Umsetzung in den einzelnen Mitgliedstaaten zwangsweise zu uneinheitlichen Standards führt. Dies zeigt sich aber auch und sogar noch verstärkt bei der Integration der Anforderungen an den guten landwirtschaftlichen und ökologischen Zustand (GLÖZ), wo verschiedene Mindestanforderungen an bestimmte Themen vorgegeben werden, die von den Mitgliedstaaten auf nationaler oder regionaler Ebene auszufüllen sind. Im Detail zu Regelungstechnik und Inhalt bspw. G. Holzer, Die neue „Ökoarchitektur “..., S. 136 ff.

${ }^{69}$ G. Holzer, Die neue „Ökoarchitektur“..., S. 143.

${ }^{70}$ R. Norer, Rechtsfragen ..., S. 125 f.; G. Holzer, Die neue „Ökoarchitektur “..., S. 141.

${ }^{71}$ So z.B. im Verhältnis Verwaltungsrecht - Zivilrecht P. Tschannen, U. Zimmerli, M. Müller, Allgemeines Verwaltungsrecht, Bern 2014, S. 130.

${ }^{72}$ R. Norer (Rechtsfragen..., S. 126), wonach zwischen der juristischen und der rechtspolitischen Seite zu unterscheiden ist. 
EuGH hat allerdings in zahlreichen Urteilen wiederholt bestätigt, dass die Agrarkompetenz des (aktuell) Art. 43 AEUV weit auszulegen ist. So hat der Gerichtshof im Fall von BSE-Maßnahmen bzw. Agrarumweltmaßnahmen in Abgrenzung zum Verbraucherschutz- bzw. Umweltkompetenzartikel die Abstützung auf die Agrarkompetenz als ausreichend befunden, da ein Rechtsakt, der zwei Zielsetzungen hat, auf die wesentliche oder überwiegende Komponente abzustützen ist ${ }^{73}$.

Ob die Implementierung von Umweltmaßnahmen in der 1. Säule überhaupt eine effiziente Erreichung von Umweltzielen ermöglicht, wird mit Hinweis auf die Pauschale Anwendung unterschiedlich beurteilt ${ }^{74}$.

\subsection{Vergleich}

Bei einer Gesamtschau aller drei hier beschriebenen Methoden erweisen sich sowohl die Trennung als auch die Integration als rechtlich weitgehend unproblematisch. Bei der Trennung vollziehen die Nicht-Agrarbehörden ihre eigenen Materien für alle davon Betroffenen (Landwirte oder nicht), bei der Integration vollziehen die Agrarbehörden ihr eigenes agrarisches Recht. Insofern handelt es sich auch bei außerlandwirtschaftlichen Einschlüssen um Agrarrecht, das von den Agrargesetzgebern nach einem politischen Kompromiss erlassen wird. D.h. es handelt sich dann kaum je um „unverfälschtes“ Umwelt-, Sozial- oder Lebensmittelrecht, sondern um eine auf die Landwirtschaft abgestimmte Fassung, die insofern in höchstem Maße geeignet sein sollte, die damit verfolgten Ziele zu erreichen.

Einzig bei der Verknüpfung zeigen sich Rechtsprobleme, wenngleich sich diese Methode seit ihrer Einführung als erstaunlich resistent gezeigt hat und immer noch zeigt. Konzeptionell erstaunt es nicht, dass es zu rechtlichen Reibepunkten kommen muss, wenn eine für einen anderen Rechtsbereich konzipierte Norm tel quel im Agrarrecht angewendet wird. Der dahinterstehende Gedanke sieht die Gewährung von Agrarförderungen als über das Agrarrecht hinausgehend von einer gesamtgesellschaftlichen Perspektive. So hätte bspw. ein Tierhalter, der verbotene Antibiotika einsetzt, zwar ,agrarfremd" ein Strafverfahren samt Geldstrafe zu vergegenwärtigen, könnte aber

${ }^{73}$ EuGH, Rs. C-269/97, Slg. 2000, I-2257; C-336/00, Slg. 2002, I-7699. Vgl. Zu Kollisionsfällen R. Norer, Art. 43 AEUV Rz. 5, in: M. Pechstein, C. Nowak, U. Häde (Hrsg.), Frankfurter Kommentar..., Band II.

${ }^{74}$ So B. Mittermüller, Die Ökoprämie und ihre ,eigentümliche“ Rechtsnatur, in: R. Norer, G. Holzer (Hrsg.), Agrarrecht Jahrbuch, Wien - Graz 2014, S. 161; B. Heinrich, C. Holst, S. Lakner, Die Reform der gemeinsamen Agrarpolitik: Wird alles grüner und gerechter?, „GAIA“ 2013, Nr. 22/1, S. 22 f.: a.A. G. Holzer, Die neue „Ökoarchitektur “..., S. 151. 
ohne Verlinkung die von der Union gewährten Direktzahlungen behalten. Dies sei der Öffentlichkeit nicht verständlich zu machen und nicht zu vereinbaren mit der staatlichen Vorbildfunktion ${ }^{75}$. Insofern läge die Einhaltung der Standards auch im Interesse der GAP selbst, da so die gesellschaftliche Akzeptanz der Agrarbeihilfen erhöht werde ${ }^{76}$.

\subsection{Ergebnis}

Aus Regulierungssicht stehen also drei Instrumente zur Verfügung. In der oben beschriebenen Ökopyramide verdichten sich derzeit alle drei Regelungstechniken. Unten die Trennung beim umweltrelevanten Fachrecht (= allgemeines Umweltrecht), dann die Verlinkung bei Cross Compliance und an der Spitze die Integration bei Greening und Agrarumweltmaßnahmen ${ }^{77}$. Diese Instrumente können nur dann erfolgreich sein, wenn jeweils bestimmte Voraussetzungen gegeben sind.

Die Trennung setzt nicht nur voraus, dass das außerlandwirtschaftliche Recht von den agrarfremden Behörden effektiv vollzogen werden kann, sondern auch ein von außerlandwirtschaftlichen Ansprüchen bereinigtes Agrarrecht. Das würde letztlich bedeuten, dass (wieder) jede Politik für sich legiferieren und vollziehen würde, die Umweltpolitik wäre die Umweltpolitik, die Strukturpolitik die Strukturpolitik, die Klimapolitik die Klimapolitik usw. mit klar abgegrenzten Kompetenzen und Budgets ${ }^{78}$. Das würde allerdings Umfang und (auch finanzielle) Bedeutung des Agrarrechts deutlich schmälern und es auf seine historischen Kernaufgaben i.S. des Art. 39 AEUV und da insbesondere die Wahrnehmung der Nahrungsfunktion zurückwerfen.

${ }^{75}$ R. Norer, Rechtsfragen..., S. 104 f. m.w.N.

${ }^{76}$ Ch. Busse, Art. 40 AEUV Rz. 79, in: C.O. Lenz, K.-D. Borchardt (Hrsg.), EU-Verträge Kommentar, Köln - Wien 2012.

${ }^{77}$ Die jüngste Reformetappe der GAP wird im hier behandelten Themenbereich insofern Änderungen bewirken, als die derzeit dreistufige Ökoarchitektur (Cross Compliance, Greening, Agrarumwelt- und Klimamaßnahmen) auf zwei Stufen reduziert wird. Dazu wird das Greening im Cross-Compliance-System unter dem neuen Titel der Ökokonditionalität aufgehen. Damit wird sich die „Baseline“ noch einmal deutlich erhöhen und der normative Geltungsgrund ändern. Darüber hinaus gehen die klima- und umweltbezogenen Zahlungen, die in beiden Säulen vorgesehen sind; R. Mögele, I.E. Rusu, Die Vorschläge der Kommission für die Gemeinsame Agrarpolitik nach 2020, „CEDR-JRL“ 2018, Nr. 2, S. 12; im Detail G. Holzer, Die neue Ökoarchitektur ..., S. 196 ff. und zuletzt idem, Ökoarchitektur der Gemeinsamen Agrarpolitik im Umbruch, in: J. Martínez (Hrsg.), Jahrbuch des Agrarrechts, Band XV, Baden-Baden 2021, S. 71 ff.

${ }^{78}$ In diese Richtung bereits R. Norer, Synthesebericht, in: C.E.D.R. (Hrsg.), CAP Reform: Market Organisation and Rural Areas. Legal Framework and Implementation. XXVIII European Congress and Colloquium of Agricultural Law, Potsdam. 9-13 september 2015, Baden-Baden 2017, S. 423 . 
Die Integration setzt nicht nur die Übernahme außerlandwirtschaftlicher Ansprüche ins Agrarrecht voraus, sondern in letzter Konsequenz auch die Ausnahme der Landwirtschaft von der Geltung des allgemeinen Rechts. Das würde eine vielfältig allen außerlandwirtschaftlichen Ansprüchen gerecht werden wollende Agrarpolitik bedeuten, die angetreten wäre, gleichsam als Superheld die Welt zu retten: seit vorgestern die Umwelt, gestern die Lebensmittelsicherheit, heute das Tierwohl, morgen das Klima und übermorgen was auch immer. Das hätte unweigerlich die Überforderung des Agrarrechts zur Folge, das Gefahr laufen würde konturlos und beliebig zu werden.

So betrachtet ist das rein rechtlich am wenigsten überzeugende Instrument, nämlich die Verknüpfung, vielleicht doch das Maximum des Machbaren zwischen agrarrechtlicher Segregation und Überforderung. Eine Weltenrettung im Kleinen mit methodischen Mängeln sozusagen.

\section{BIBLIOGRAPHIE}

C.E.D.R. (Hrsg.) (2017), CAP Reform: Market Organisation and Rural Areas. Legal Framework and Implementation. XXVIII European Congress and Colloquium of Agricultural Law, Potsdam. 9-13 september 2015, Baden-Baden.

Eckhardt G. (2010), Die Reform der GAP 2003 - Zwischenbilanz und Ausblick, in: R. Norer, G. Holzer (Hrsg.), Agrarrecht Jahrbuch, Wien - Graz.

Grimm Ch., Norer R. (2015), Agrarrecht, München.

Groeben H. von der, Boeckh H. von, Thiesing J., Ehlermann C.D. (Hrsg.) (1983), Kommentar zum EWG-Vertrag, Baden-Baden.

Heinrich B., Holst C., Lakner S. (2013), Die Reform der gemeinsamen Agrarpolitik: Wird alles grüner und gerechter?, „GAIA“ Nr. 22/1.

Holzer G. (2015), Die neue „Ökoarchitektur“ der GAP, in: R. Norer, G. Holzer (Hrsg.), Agrarrecht Jahrbuch, Wien - Graz.

Holzer G. (2019), Die neue Ökoarchitektur der GAP und ihr Beitrag zum Klimaschutz, in: R. Norer, G. Holzer (Hrsg.), Agrarrecht Jahrbuch, Wien - Graz.

Holzer G. (2021), Ökoarchitektur der Gemeinsamen Agrarpolitik im Umbruch, in: J. Martínez (Hrsg.), Jahrbuch des Agrarrechts, Band XV, Baden-Baden.

Lenz C.O., Borchardt K.-D. (Hrsg.) (2012), EU-Verträge Kommentar, Köln - Wien.

Martínez J. (2013), Das Greening der Gemeinsamen Agrarpolitik, „Natur und Recht“"Nr. 35.

Mittermüller B. (2014), Die Ökoprämie und ihre ,, eigentümliche“ Rechtsnatur, in: R. Norer, G. Holzer (Hrsg.), Agrarrecht Jahrbuch, Wien - Graz.

Mögele R., Rusu I.E. (2018), Die Vorschläge der Kommission für die Gemeinsame Agrarpolitik nach 2020, „CEDR-JRL“ Nr. 2.

Norer R. (2007), Rechtsfragen der EU-Agrarreform, Wien - Graz.

Norer R. (2020), Die Zukunft der Gemeinsamen Agrarpolitik: Europarechtlicher Trendsetter oder Expertenhochburg?, in: R. Norer, G. Holzer (Hrsg.), Agrarrecht Jahrbuch, Wien.

Pechstein M., Nowak C., Häde U. (Hrsg.) (2017), Frankfurter Kommentar EUV, GRC und $A E U V$, Band II, Tübingen. 
Peine F.-J. (2005), Verknüpfung der Beihilfen mit der Einhaltung von Umweltstandards Konsequenzen, „Agrar- und Umweltrecht“ Beilage I.

Queisner G. (2013), Rahmenbedingungen für eine umweltverträgliche Landwirtschaft im Europarecht. Zugleich ein Beitrag zur Reform der GAP, Cross Compliance und Klimaschutz, Baden-Baden.

Schwarze J. (2005), Europäisches Verwaltungsrecht, Baden-Baden.

Tschannen P., Zimmerli U., Müller M. (2014), Allgemeines Verwaltungsrecht, Bern.

\title{
AGRICULTURAL LAW SAVING THE WORLD, OR MAKING TOO HIGH DEMANDS? ON THE METHODOLOGY FOR INTEGRATING NON-AGRICULTURAL CLAIMS INTO EUROPEAN AGRICULTURAL LAW
}

\author{
Summary
}

In the course of its development, starting with the organisation of agricultural markets, the Common Agricultural Policy has been confronted with many different, also non-agricultural problems. It had to respond to the requirements of environmental protection, structural and social policy, animal welfare, energy policy or climate protection. Nowadays, there are three different methods by which the relationship between agricultural law and non-agricultural legal issues is legally shaped: separation, connection and integration.

Keywords: Common Agricultural Policy, environmental policy, structural policy, legal methodology, cross-compliance, greening

\section{SALVARE IL MONDO GRAZIE AL DIRITTO AGRARIO DI FRONTE A RICHIESTE TROPPO ELEVATE... SULLA METODOLOGIA DI INCORPORARE LE RIVENDICAZIONI NON CONNESSE ALL'AGRICOLTURA NEL DIRITTO AGRARIO EUROPEO}

\section{Riassunto}

La politica agricola comune, nelle diverse fasi di sviluppo, a partire da quella di organizzare i mercati agricoli, ha dovuto affrontare problemi diversi, non sempre connessi all'agricoltura. È stata costretta a soddisfare i requisiti in ambito di protezione ambientale, politica strutturale e sociale, benessere degli animali, politica energetica oppure tutela del clima. Al giorno d'oggi si possono osservare tre diversi metodi attraverso i quali si configura, dal punto di vista giuridico, il rapporto tra diritto agrario e materia giuridica non connessa all'agricoltura: separazione, relazione e integrazione.

Parole chiave: politica agricola comune, politica ambientale, politica strutturale, metodologia giuridica, cross-compliance, greening 